\title{
Pulmonary Hypertension Associated With HIV Infection
}

\section{Pulmonary Vascular Disease: The Global Perspective}

\author{
Sharilyn Almodovar, PhD; Stefania Cicalini, MD; Nicola Petrosillo, MD; \\ and Sonia C. Flores, PhD
}

\begin{abstract}
The success of antiretroviral therapies in improving the survival of patients infected with HIV and reducing HIV-associated opportunistic infections is undisputed. Nevertheless, long-term outcomes such as noninfectious cardiovascular complications, including cardiomegaly, pericarditis, myocarditis, and pulmonary arterial hypertension, are now serious concerns. The lung is a frequent target organ for disorders associated with HIV infection. HIV-related pulmonary arterial hypertension (HRPAH) affects more individuals who are infected with HIV than individuals who are uninfected. Moreover, the long-standing estimated prevalence of HRPAH in developed countries (calculated at $0.5 \%$ ) is increasing as more clinician-scientists unify their efforts to screen patients who are pulmonary asymptomatic for pulmonary arterial hypertension. In order to decrease mortality, efforts are directed at early detection, diagnosis, and therapeutic interventions before the disease compromises patients' quality of life. This article reviews the logistics of screening approaches for HRPAH and discusses the substantial disease burden currently faced by developing countries, where the prevalence of HIV infection is higher and complicated by hyperendemic risk factors, limited access to antiretrovirals, and lack of screening tools. We also present mechanistic insights into HRPAH, including the role of HIV proteins and their potential use as screening tools, and, finally, areas that still need intense research. CHEST 2010; 137(6)(Suppl):6S-12S

Abbreviations: $\mathrm{ART}=$ antiretroviral therapy; $\mathrm{BMPR} 2=$ bone morphogenetic protein receptor type 2; Env $=$ envelope glycoprotein-120; HRPAH $=$ HIV-related pulmonary arterial hypertension; $n e f=$ negative factor; $\mathrm{PAH}=$ pulmonary arterial hypertension; RHC = right-sided heart catheterization; SHIVnef = simian-human immunodeficiency virus negative factor; Tat $=$ transactivator of transcription
\end{abstract}

$\mathbf{C}$ ardiac involvement and cardiopulmonary-vascular

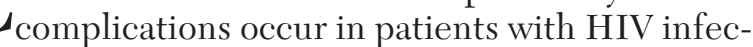
tion and include pericardial effusion, myocarditis, dilated cardiomyopathy, endocarditis, coronary artery disease, malignant neoplasms, and pulmonary arterial hypertension (PAH). Patients who are infected with HIV and have HIV-related PAH (HRPAH) exhibit increased mortality compared with counterparts who are HIV-positive and normotensive; half of the deaths reported in patients with HIV infection as the only risk factor for PAH are due to cardiopulmonary complications (eg, right ventricular failure or sudden cardiac death) and not to HIV/AIDS itself. ${ }^{1}$ Approximately 90\% of the patients with HRPAH survive 1 year, while $70 \%$ have a 3 -year survival rate. ${ }^{1,2}$ This article focuses on HRPAH, with special considerations to prevalence, clinical presentation and diagnosis in the developing world, and the need for more intense research.

\section{PAH AS A LONG-TERM COMPLiCATION OF HIV INFECTION: WHAT IS KNOWN?}

The antiretroviral cocktails introduced more than one decade ago have significantly improved survival for patients infected with HIV and reduced HIVrelated opportunistic infections. However, 2 million lives were claimed by this infection in $2008,{ }^{3}$ although deaths due to noninfectious complications of HIV infection are not accounted for in these estimates. Long-term outcomes, such as cardiovascular complications, including cardiomegaly, pericarditis, myocarditis, and PAH secondary to HIV infection, are now serious concerns. ${ }^{4}$ 
The lung is a frequent target organ for disorders associated with HIV infection. PAH is diagnosed more frequently in the patient population infected with HIV than in the general population., ${ }^{1,2}$ The longstanding estimated prevalence of HRPAH in developed countries is $0.5 \% .^{2,5-8}$ As much as $27 \%$ of patients with suspected PAH by echocardiographic screening were diagnosed with HRPAH via right-sided heart catheterization (RHC). ${ }^{7}$ Based solely on echocardiographic parameters, $35 \%$ of individuals infected with HIV have been identified with "preclinical" $\mathrm{PAH},{ }^{9}$ and $5.5 \%$ of patients who are asymptomatic may be at risk for PAH. ${ }^{10}$ Comprehensive longitudinal studies have reported that the average age of patients with HRPAH is 38 years in a Swiss HIV study ${ }^{2}$ and 41 in a French study, ${ }^{1}$ although the range can span from infancy to old age. Men are slightly more affected by HRPAH than women (ratio of 1.2:1,2,11). There is no statistically significant association between PAH and viral loads or $\mathrm{CD}^{+}{ }^{+} \mathrm{T}$-cell counts, ${ }^{11,12}$ but $\mathrm{PAH}$ is more severe in patients with AIDS. ${ }^{1,13}$ The role of antiretroviral therapy (ART) in PAH is still being debated; some studies suggest that HRPAH develops regardless of ART, ${ }^{14}$ that hemodynamic parameters by RHC remain unchanged, ${ }^{1}$ or that ART may accelerate the onset of HRPAH disease. ${ }^{15}$ On the other hand, other studies consider that therapy increases the survival ${ }^{2}$ and decreases the prevalence of cardiovascular diseases. In addition, there is no etiologic connection between HRPAH and the familial form of $\mathrm{PAH}^{11,16}$ ascribed to germ-line mutations in bone morphogenetic protein receptor type 2 (BMPR2).

\section{The Clinical Framework for HRPAH}

Patients with HRPAH are frequently misdiagnosed. Shortness of breath is the most common symptom, and although it is often ascribed to deconditioning,

Manuscript received December 21, 2009; revision accepted February 13, 2010.

Affiliations: From the Cardiovascular Pulmonary Laboratory and Department of Pulmonary Sciences and Critical Care Medicine (Drs Almodovar and Flores), University of Colorado-Denver, Aurora, CO; and the Second Infectious Diseases Unit (Drs Cicalini and Petrosillo), National Institute for Infectious Diseases, Lazzaro Spallanzani, Istituto di Ricovero e Cura a Carattere Scientifico, Rome, Italy. Drs Almodovar and Cicalini equally contributed to this manuscript.

Funding/support: A portion of this study was supported by the National Institutes of Health/National Heart, Lung, and Blood Institute [Grants R01 HL083491, R01 HL90480, and HL07171]. Correspondence to: Sharilyn Almodovar, PhD, University of Colorado Denver, Pulmonary Sciences and Critical Care Medicine, 12700 E 19th Ave, C-272, Aurora, CO 80045; e-mail: Sharilyn.Almodovar@ucdenver.edu

(C) 2010 American College of Chest Physicians. Reproduction of this article is prohibited without written permission from the American College of Chest Physicians (http://www.chestpubs.org/ site/misc/reprints.xhtml).

DOI: $10.1378 /$ chest.09-3065 it usually progresses to the point of breathlessness at rest. ${ }^{17}$ Other symptoms include pedal and peripheral edema, nonproductive cough, fatigue, and chest pain. Right ventricular enlargement becomes evident by chest radiography and transthoracic echocardiography. The assessment of hemodynamic measures by cardiac catheterization is the gold standard for the diagnosis of $\mathrm{PAH}$ and the best test for evaluating the response to therapy. Cardiac catheterization, however, is not available in most health-care centers, including tertiary hospitals in sub-Saharan Africa and many other parts of the developing world. ${ }^{18}$

Overall, HRPAH is typically diagnosed late in its course, when the clinical and laboratory findings of severe $\mathrm{PAH}$ (eg, right ventricular dysfunction) are generally present. ${ }^{17}$ Therefore, early detection is essential for the diagnosis, and clinical interventions are needed before quality of life is compromised.

\section{HRPAH Is a Medical Problem With UnMeT NeEds WorLdWide: ITS Potential IMPACT ON THE DEVELOPING WORLD}

Unfortunately, Africa has the largest number of inhabitants with HIV/AIDS worldwide $(\sim 23$ of the 33 million people infected). Global efforts have yielded a 10-fold increase in availability of ART over 5 years; $43 \%$ of the African population now has access to HIV treatments. ${ }^{3}$ Still, pericardial and myocardial diseases associated with opportunistic infections prevail in $20 \%$ to $60 \%$ of the patients, according to pathologic and diagnostic methods and evaluation of the study population. Evidence of PAH in Africa is indirect because studies have been limited to echocardiographic measurements and anecdotes from African individuals. Echocardiographic abnormalities suggestive of $\mathrm{PAH}$ were found in $0.6 \%$ to $5 \%$ of the patients infected with HIV in Burkina Faso and Zimbabwe. ${ }^{19}$ Echocardiographic suspicions of PAH should be placed in the context of the relatively higher prevalence of pulmonary diseases (eg, TB, bronchiectasis, interstitial lung diseases), comorbidities (eg, sickle cell anemia, malnutrition, wasting syndrome, liver cirrhosis), and coinfections that are more prevalent in Africa. Cardiopulmonary pathologic conditions related to HIV infection are complicated by several additional infectious risk factors for PAH that are hyperendemic in regions like sub-Saharan Africa; these include schistosomiasis, filariasis, malaria, and chronic hepatitis B and C. In addition, human herpes virus 8, which is endemic in South America and subSaharan Africa, can be found in $62 \%$ of cells within and around the plexiform lesions in the lungs. ${ }^{20}$ Anecdotal data from centers in Africa indicate little difference between the clinical manifestations of the disease 
in Africa and the developed world. ${ }^{21}$ The fact that there are no established population studies focused on HRPAH in the African countries does not preclude judicious extrapolations from the developed world. Even if the percentage of people living with HIV who develop clinically defined HRPAH is relatively small, the disease burden in developing countries could be unbearable in the face of the high prevalence of HIV infection in these regions.

\section{Ongoing Research Initiatives DiRected AT A BETTER UNDERSTANDING OF HRPAH}

To understand any disease, cohorts of patients need to be characterized and followed up closely. The Swiss HIV Cohort Study, which followed patients for more than two decades, pioneered the determination of cumulative prevalence of HRPAH. ${ }^{6}$ The French National Registry of PAH has standardized the diagnosis of PAH by adopting an algorithm that includes selfreported dyspnea followed by echocardiographies and right-sided heart catheterization if there is clinical suspicion of $\mathrm{PAH}$ (eg, dyspnea in the absence of heart/ lung diseases). Although not confirmed by RHC, echocardiography-based studies have focused the spotlight on subsets of patients in different cohorts worldwide. Some of these studies, such as the Swiss HIV Cohort Study, the French HIV-PAH Registry, and the Latium Registry of HRPAH, monitor the patients prospectively. Similarly, the National Heart, Lung, and Blood Institute of the National Institutes of Health in the United States has recently initiated a similar eightsite effort to understand clinically overlapping pulmonary topics related to HIV infection, including PAH, COPD, bacterial pneumonias, and emphysema.

\section{MECHANISTIC Insights INTO HRPAH}

The histopathologic characteristics of HRPAH are not different from those of idiopathic PAH. ${ }^{17}$ Briefly, the pulmonary vasculature is obliterated with medial hypertrophy and increased proliferation of endothelial and smooth muscle cells. Plexiform lesions can be detected in $78 \%$ of patients with HRPAH. ${ }^{17}$ There is increased expression of smooth muscle cell/fibroblast growth factors such as platelet-derived growth factor. ${ }^{22}$ Inflammatory cells (eg, macrophages, lymphocytes, and dendritic cells) in the perivasculature in patients with severe primary $\mathrm{PAH}$ point to a potential role of inflammation in $\mathrm{PAH},{ }^{23,24}$ which is truly more evident in HRPAH. Essentially, HIV is at the center of the loop of PAH inflammation because the chronic inflammation and immune activation produced by HIV infection may lead to increased secretion of proinflammatory cytokines and growth factors that may subsequently promote $\mathrm{PAH}$.

Besides the inflammatory aspect of HRPAH, Sehgal and Mukhopadhyay ${ }^{25}$ presented an alternative view that suggests that disrupted subcellular membrane trafficking in endothelial cells and smooth muscle cells is critical in the pathobiologic aspect of PAH. Observations of endothelial cells in plexiform lesions by electron microscopy revealed plump cells with enlarged endoplasmic reticulum, Golgi stacks, and vacuolation, ${ }^{26}$ suggesting defects in intracellular trafficking. Recent studies showed aberrant subcellular distribution of Golgi tethers giantin and p115 in obliterativeplexiform lesions in patients with idiopathic $\mathrm{PAH}$ and in macaques infected with simian-human immunodeficiency virus negative factor (SHIVnef). ${ }^{27}$ The amounts of Golgi tethers/matrix proteins were increased on a per-cell basis; together, these results suggest that subcellular trafficking and Golgi dysfunction may have important roles in the development of HRPAH.

\section{HIV Plays a Role in the Ethiopathology of PAH}

There is no definitive evidence that HIV is a causal agent for PAH. Whether HIV infects lung vascular endothelial cells is still under discussion. ${ }^{22,28}$ The virus itself has not been detected in the lesions exhibited by patients with HRPAH.22,29 However, viral proteins and their interactions with molecular partners in the infected host are strong candidates for cause-effect relationships because they may promote apoptosis, growth, and proliferation. ${ }^{29}$ Pulmonary hypertension has been associated with viral proteins in infections with hepatitis $\mathrm{B}^{30}$ and human herpes virus- $8,{ }^{20}$ although the latter is not statistically associated with PAH.9,31

HIV proteins that damage endothelial cells and induce inflammation may result in pulmonary vascular remodeling. For example, the HIV envelope glycoprotein-120 (Env), which is essential for viral attachment and fusion through the host cellular membrane, induces apoptosis and increases the secretion of endothelin-1 from human lung endothelial cells..$^{28}$ Also, HIV Env stimulates monocytes and macrophages to release proinflammatory cytokines. ${ }^{32}$ The HIV transactivator of transcription (Tat) protein activates endothelial cells and has angiogenic properties..$^{33}$ Even though mutations in BMPR2 are not associated with HRPAH, the finding that Tat represses BMPR2 in monocytes ${ }^{34}$ suggests that idiopathic PAH might be biologically linked (not etiologically) to HRPAH.

HIV negative factor (Nef) is an accessory protein expressed early during viral infection, together with Tat. The Nef protein is a molecular adaptor and is a key player in HIV pathogenesis. For example, Nef interacts with several host cell proteins (including members of the p21-activated kinase family $)^{35}$ and 


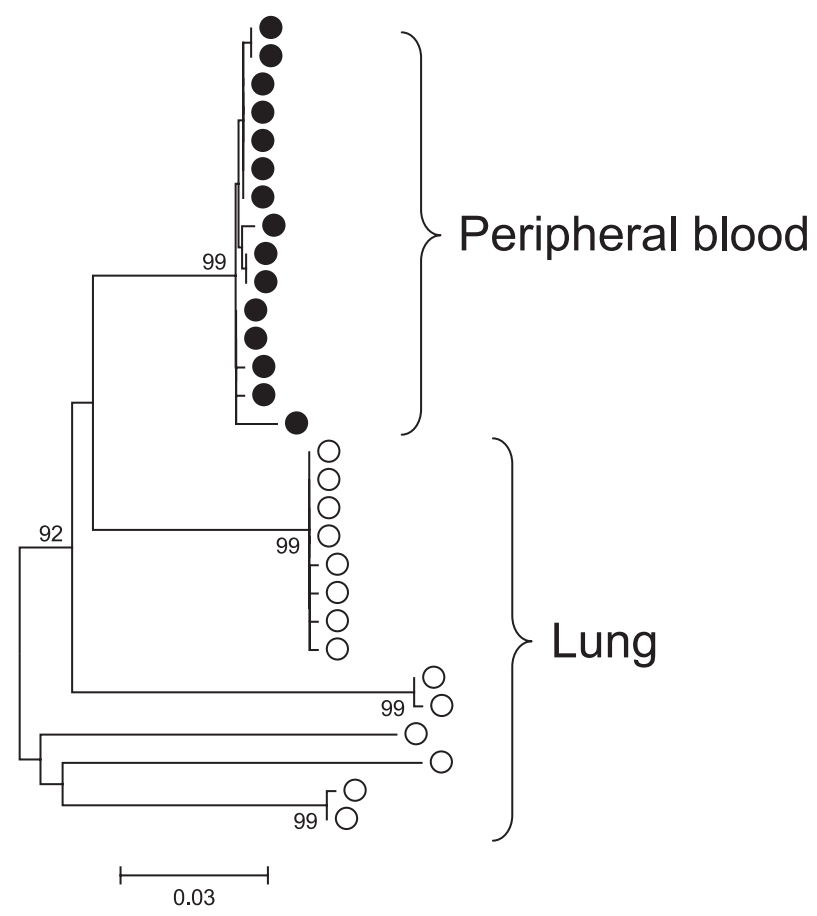

FIGURE 1. Phylogenetic reconstruction of HIV-1 negative factor (nef) sequences from a patient diagnosed with HIV-related pulmonary arterial hypertension (HRPAH). Archived peripheral blood mononuclear cells and lung tissue were obtained by echocardiography and enrolled in the Latium Registry of HRPAH in Rome. HIV nef was amplified using polymerase chain reaction, cloned, and sequenced; sequences were aligned using BioEdit (Ibis Therapeutics; Carlsbad, CA), and the phylogenetic tree was created using MEGA4 (The Biodesign Institute; Tempe, AZ). The analysis was statistically supported by 1,000 bootstrap resamplings, and only values $>70 \%$ are shown. Tissue-specific clustering of the nef quasi-species suggests differential evolution at the level of $\operatorname{blood}(\mathbf{O})$ and lung $(\bigcirc)$.

recruits host adaptor proteins to commandeer trafficking of intracellular vesicles participating in secretory and endocytic pathways. ${ }^{36}$ Nef downregulates essential molecules such as the CD4 receptor by targeting the endocytic degradation pathway in clathrin-coated vesicles and major histocompatibility complex type 1 by sequestering it in the trans-Golgi and preventing its recycling from the Golgi to the membrane. ${ }^{37}$ Also, Nef is a migratory stimulus for monocytes $^{38}$ and induces the release of inflammatory molecules. ${ }^{39}$ Capoccia and colleagues ${ }^{40}$ proposed a model in which Nef induces early recruitment of inflammatory monocytes, which in turn induce a secondary wave of monocytes followed by angiogenesis.

The potential role of Nef as a vascular insult is underscored by its capacity to enter lymphocytes via the human chemokine (C-X-C motif) receptor 4 (fusin) and exert apoptotic effects. ${ }^{41}$ Endothelial cells express this receptor, and therefore it is conceivable that Nef may be present in endothelial cells in the absence of infection. Nef localizes to vascular and perivascular cells ${ }^{42,43}$ and induces apoptosis in brain endothelial cells when expressed intracellularly or exogenously. ${ }^{44}$
Recently, Lenassi and colleagues ${ }^{45}$ showed that nef-infected cells secrete Nef-containing exosomes that in turn induce apoptosis in resting $\mathrm{T}$ lymphocytes in vitro. Nef also impairs vasomotor functions in pulmonary artery cells, decreases the expression of endothelial nitric oxide synthase, and increases oxidative stress. ${ }^{46}$

The use of chimeric viruses has facilitated the study of specific HIV proteins in animal models. For example, SHIVnef is a chimeric virus that preserves the backbone of simian immunodeficiency virus, the simian counterpart of HIV, but the nef gene was replaced for the HIV nef recovered from a patient with AIDS. ${ }^{47}$ Rhesus macaques infected with SHIVnef developed simian AIDS. Interestingly, Marecki and colleagues ${ }^{42}$ observed vascular remodeling in the lungs of rhesus macaques infected with SHIVnef that was similar to the remodeling exhibited by patients with PAH. Furthermore, the same research group reported plexiform lesions exclusively in banked lung samples from macaques experimentally infected with SHIVnef, but not in macaques infected with simian immunodeficiency virus. ${ }^{43}$ The plexiform-like lesions were characterized by luminal obliteration, endothelial cell proliferation, medial hypertrophy, thrombosis, and recanalized lumina. These data strongly suggest a role for Nef in the formation of plexiform lesions in the lung vasculature.

\section{Is There a Diagnostic Marker of HRPAH?}

The high mutational profile of HIV is the main hurdle in the design and development of vaccines to prevent and/or cure this infection. However, this feature of HIV may set the stage for the selection of specific HIV quasi-species at specific anatomic sites where virus presence may translate into a disease phenotype.

\section{Nef Is Mutated in the Lung of a Patient With HRPAH}

Nef sequences in the blood of patients with pulmonary hypertension show signature patterns that are specific to this disease phenotype, compared with their normotensive counterparts. ${ }^{48}$ Peripheral blood samples are the main biologic specimens analyzed in clinical research because lung tissues from these patients are extremely difficult to obtain. Nevertheless, Petrosillo et al were able to amplify and clone HIV-1 nef sequences from archived lung tissue from a patient with HRPAH from the Italian Latium Registry of HRPAH. ${ }^{49}$ The patient received a diagnosis of HIV infection in 2000 and of HRPAH in 2006, the same year the patient died. The lungs were collected 


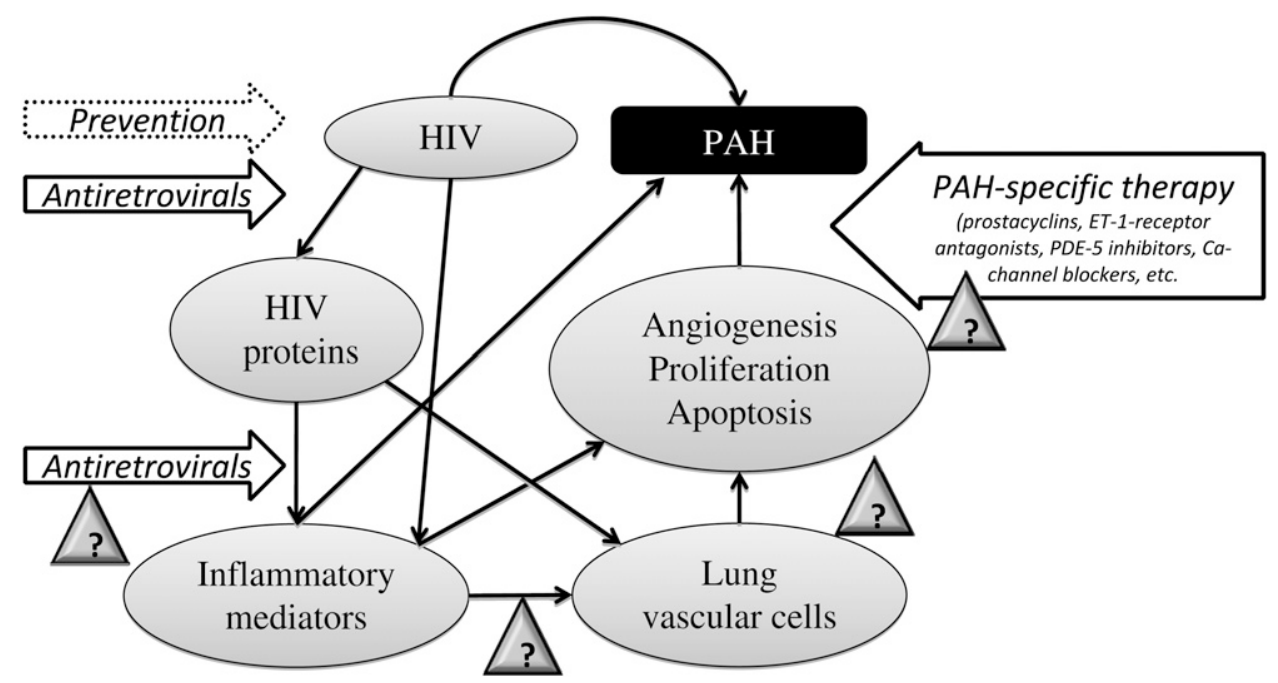

Figure 2. Conceptual frame of PAH as a complication of HIV infection. The connections between hypothetical events contributing to HRPAH are indicated with black arrows. Therapeutic interventions are shown by the white block arrows. Areas that warrant further research to determine molecular mechanisms and potentially unravel future therapeutic targets are indicated by triangles. $\mathrm{PAH}=$ pulmonary arterial hypertension. See Figure 1 for expansion of the other abbreviation.

during autopsy and subsequently analyzed. Sequence analyses of the HIV nef showed the presence of $4 / 5$ Nef mutations reported in macaques infected with SHIVnef ${ }^{47}$ with plexiform lesions. Furthermore, this Nef sequence had six Nef functional domains mutated, all of them found only in the lung tissues collected in 2006, and interestingly, none of the mutations was found in peripheral blood mononuclear cells collected at the time of HIV diagnosis. There are clear phylogenetic differences between the quasi-species of the lung vs the periphery (Fig 1). These findings suggest either that the viral quasispecies in the lung arose later in the course of infection or that these variants are replicating more efficiently in the lung than in the periphery. These aspects warrant further research efforts in order to validate (and possibly implement) the use of Nef sequences as potential screening tools to identify subjects at risk for HRPAH, especially in a setting where resources are scarce.

\section{ConClusion}

Although a tight association between HIV infection and coinfections with viruses or bacterial agents in HRPAH remains to be established, interactions between HIV and other infectious diseases may result in an even higher prevalence of $\mathrm{PAH}$ in patients infected with HIV and influence the natural history, treatment, and outcome of HRPAH in Africa and other developing countries. Hence, increased awareness in the medical communities worldwide is an important step forward in understanding and treating this disease. Although there is no direct evidence that HIV causes PAH, it is generally accepted among the clinical-scientific community that HRPAH is more likely to occur via indirect action of HIV. Because HIV-infected cells in the lungs are sources of HIV proteins, the chronic exposure of lung endothelial cells to viral proteins has been hypothesized as one of the most important reasons for lung vascular injury in patients infected with HIV. The HIV proteins Env, Tat, and Nef have been identified as offenders of the pulmonary vasculature and, hence, key players in the pathogenesis of HRPAH. However, the understanding of the cellular and/or subcellular mechanisms and the pathways involved will be critical in unraveling the role of HIV proteins in HRPAH and research areas that need more attention (Fig 2).

The detection of elevated pulmonary artery pressures in patients infected with HIV who are asymptomatic for pulmonary complications denotes that a considerable number of these patients may be at risk for developing HRPAH and/or are exhibiting preclinical signs of HRPAH. This increases the urgency for the identification of biomarkers that will predict the likelihood of $\mathrm{PAH}$ in patients who are asymptomatic and in those with unexplained cardiopulmonary symptoms. A wealth of data suggests that HIV-1 Nef is a broad-spectrum modulator that may impact both infected and uninfected cells. Thus far, the potential use of mutations in Nef as a screening tool for HRPAH opens the door for further studies focused on the consequences of such mutations in HIV Nef in the context of pulmonary vascular biology. 


\section{ACKNOWLEDGMENTS}

Financial/nonfinancial disclosures: The authors have reported to CHEST the following conflicts of interest: Dr Petrosillo has received speaker's honoraria from Sanofi Pasteur MSD Limited, Janssen Cilag, Astellas Pharma, Pfizer, Novartis, Astra Zeneca, and GlaxoSmithKline. Drs Almodovar, Cicalini, and Flores have reported that no potential conflicts exist with any companies/ organizations whose products or services may be discussed in this article.

\section{REFERENCES}

1. Degano B, Guillaume M, Savale L, et al. HIV-associated pulmonary arterial hypertension: survival and prognostic factors in the modern therapeutic era. AIDS. 2010;24(1):67-75.

2. Zuber JP, Calmy A, Evison JM, et al; Swiss HIV Cohort Study Group. Pulmonary arterial hypertension related to HIV infection: improved hemodynamics and survival associated with antiretroviral therapy. Clin Infect Dis. 2004;38(8):1178-1185.

3. Joint United Nations Programme on HIV/AIDS (UNAIDS) and World Health Organizations (WHO). AIDS Epidemic Update 2009. Geneva, Switzerland: WHO; 2009.

4. Lederman MM, Sereni D, Simonneau G, Voelkel NF. Pulmonary arterial hypertension and its association with HIV infection: an overview. AIDS. 2008;22(Suppl 3):S1-S6.

5. Cicalini S, Chinello P, Cicini MP, Petrosillo N. Pulmonary arterial hypertension and HIV infection. AIDS. 2008;22(16): 2219-2220.

6. Speich R, Jenni R, Opravil M, Pfab M, Russi EW. Primary pulmonary hypertension in HIV infection. Chest. 1991; 100(5):1268-1271.

7. Sitbon O, Lascoux-Combe C, Delfraissy JF, et al. Prevalence of HIV-related pulmonary arterial hypertension in the current antiretroviral therapy era. Am J Respir Crit Care Med. 2008; 177(1):108-113.

8. Opravil M, Sereni D. Natural history of HIV-associated pulmonary arterial hypertension: trends in the HAART era. AIDS. 2008;22(Suppl 3):S35-S40.

9. Hsue PY, Deeks SG, Farah HH, et al. Role of HIV and human herpesvirus- 8 infection in pulmonary arterial hypertension. AIDS. 2008;22(7):825-833.

10. Byers DK, Nayak G, Ferguson M. Prevalence of pulmonary hypertension in asymptomatic HIV-infected patients receiving antiretroviral therapy. Paper presented at: 48th Annual International Conference on Antimicrobial Agents and Chemotherapy (ICAAC); October 25-28, 2008; Washington DC.

11. Nunes H, Humbert M, Sitbon O, et al. Prognostic factors for survival in human immunodeficiency virus-associated pulmonary arterial hypertension. Am J Respir Crit Care Med. 2003;167(10):1433-1439.

12. Humbert M, Sitbon O, Chaouat A, et al. Pulmonary arterial hypertension in France: results from a national registry. Am J Respir Crit Care Med. 2006;173(9):1023-1030.

13. Pellicelli AM, Barbaro G, Palmieri F, et al. Primary pulmonary hypertension in HIV patients: a systematic review. Angiology. 2001;52(1):31-41.

14. Pellicelli AM, D’Ambrosio C, Vizza CD, et al. HIV-related pulmonary hypertension. From pathogenesis to clinical aspects. Acta Cardiol. 2004;59(3):323-330.

15. Reinsch N, Buhr C, Krings P, et al; Competence Network of Heart Failure. Effect of gender and highly active antiretroviral therapy on HIV-related pulmonary arterial hypertension: results of the HIV-HEART Study. HIV Med. 2008;9(7): 550-556.

16. Humbert M. Mediators involved in HIV-related pulmonary arterial hypertension. AIDS. 2008;22(Suppl 3):S41-S47.
17. Mehta NJ, Khan IA, Mehta RN, Sepkowitz DA. HIV-related pulmonary hypertension: analytic review of 131 cases. Chest. 2000;118(4):1133-1141.

18. Aliyu ZY, Kato GJ, Taylor J VI, et al. Sickle cell disease and pulmonary hypertension in Africa: a global perspective and review of epidemiology, pathophysiology, and management. Am J Hematol. 2008;83(1):63-70.

19. Ntsekhe M, Hakim J. Impact of human immunodeficiency virus infection on cardiovascular disease in Africa. Circulation. 2005;112(23):3602-3607.

20. Cool CD, Rai PR, Yeager ME, et al. Expression of human herpesvirus 8 in primary pulmonary hypertension. $N$ Engl J Med. 2003;349(12):1113-1122.

21. Ntsekhe M, Mayosi BM. Cardiac manifestations of HIV infection: an African perspective. Nat Clin Pract Cardiovasc Med. 2009;6(2):120-127.

22. Humbert M, Monti G, Fartoukh M, et al. Platelet-derived growth factor expression in primary pulmonary hypertension: comparison of HIV seropositive and HIV seronegative patients. Eur Respir J. 1998;11(3):554-559.

23. Tuder RM, Groves B, Badesch DB, Voelkel NF. Exuberant endothelial cell growth and elements of inflammation are present in plexiform lesions of pulmonary hypertension. Am J Pathol. 1994;144(2):275-285.

24. Dorfmüller P, Perros F, Balabanian K, Humbert M. Inflammation in pulmonary arterial hypertension. Eur Respir J. 2003;22(2):358-363.

25. Sehgal PB, Mukhopadhyay S. Dysfunctional intracellular trafficking in the pathobiology of pulmonary arterial hypertension. Am J Respir Cell Mol Biol. 2007;37(1):31-37.

26. Smith P, Heath D. Electron microscopy of the plexiform lesion. Thorax. 1979;34(2):177-186.

27. Sehgal PB, Mukhopadhyay S, Patel K et al. Golgi dysfunction is a common feature in idiopathic human pulmonary hypertension and vascular lesions in SHIV-nef-infected macaques. Am J Physiol Lung Cell Mol Physiol 2009;297(4): L729-L737.

28. Kanmogne GD, Primeaux C, Grammas P. HIV-1 gp120 proteins alter tight junction protein expression and brain endothelial cell permeability: implications for the pathogenesis of HIV-associated dementia. J Neuropathol Exp Neurol. 2005;64(6):498-505.

29. Mette SA, Palevsky HI, Pietra GG, et al. Primary pulmonary hypertension in association with human immunodeficiency virus infection. A possible viral etiology for some forms of hypertensive pulmonary arteriopathy. Am Rev Respir Dis. 1992;145(5):1196-1200.

30. Lee SW, Lee YM, Bae SK, Murakami S, Yun Y, Kim KW. Human hepatitis B virus $\mathrm{X}$ protein is a possible mediator of hypoxia-induced angiogenesis in hepatocarcinogenesis. Biochem Biophys Res Commun. 2000;268(2):456-461.

31. Montani D, Marcelin AG, Sitbon O, Calvez V, Simonneau G, Humbert M. Human herpes virus 8 in HIV and non-HIV infected patients with pulmonary arterial hypertension in France. AIDS. 2005; 19(11):1239-1240.

32. Kim J, Ruff M, Karwatowska-Prokopczuk E, et al. HIV envelope protein gp120 induces neuropeptide Y receptormediated proliferation of vascular smooth muscle cells: relevance to AIDS cardiovascular pathogenesis. Regul Pept. 1998;75-76:201-205.

33. Rusnati M, Presta M. HIV-1 Tat protein and endothelium: from protein/cell interaction to AIDS-associated pathologies. Angiogenesis. 2002;5(3):141-151.

34. Caldwell RL, Gadipatti R, Lane KB, Shepherd VL. HIV-1 TAT represses transcription of the bone morphogenic protein receptor-2 in U937 monocytic cells. J Leukoc Biol. 2006;79(1):192-201. 
35. Fackler OT, Lu X, Frost JA, et al. p21-Activated kinase 1 plays a critical role in cellular activation by Nef. Mol Cell Biol. 2000;20(7):2619-2627.

36. Roeth JF, Collins KL. Human immunodeficiency virus type 1 Nef: adapting to intracellular trafficking pathways. Microbiol Mol Biol Rev. 2006;70(2):548-563.

37. Miller MD, Warmerdam MT, Gaston I, Greene WC, Feinberg MB. The human immunodeficiency virus-1 nef gene product: a positive factor for viral infection and replication in primary lymphocytes and macrophages. J Exp Med. 1994;179(1): 101-113.

38. Lehmann MH, Walter S, Ylisastigui L, et al. Extracellular HIV-1 Nef increases migration of monocytes. Exp Cell Res. 2006;312(18):3659-3668.

39. Olivetta E, Percario Z, Fiorucci G, et al. HIV-1 Nef induces the release of inflammatory factors from human monocyte/ macrophages: involvement of Nef endocytotic signals and NF-kappa B activation. J Immunol. 2003;170(4):1716-1727.

40. Capoccia BJ, Gregory AD, Link DC. Recruitment of the inflammatory subset of monocytes to sites of ischemia induces angiogenesis in a monocyte chemoattractant protein1-dependent fashion. J Leukoc Biol. 2008;84(3):760-768.

41. James CO, Huang MB, Khan M, Garcia-Barrio M, Powell MD, Bond VC. Extracellular Nef protein targets CD4+ T cells for apoptosis by interacting with CXCR4 surface receptors. J Virol. 2004;78(6):3099-3109.

42. Marecki J, Cool C, Voelkel N, Luciw P, Flores S. Evidence for vascular remodeling in the lungs of macaques infected with simian immunodeficiency virus/HIV NEF recombinant virus. Chest. 2005;128(6 Suppl):621S-622S.

43. Marecki JC, Cool CD, Parr JE, et al. HIV-1 Nef is associated with complex pulmonary vascular lesions in SHIV-nef-infected macaques. Am J Respir Crit Care Med. 2006;174(4):437-445.

44. Acheampong EA, Parveen Z, Muthoga LW, Kalayeh M, Mukhtar M, Pomerantz RJ. Human immunodeficiency virus type $1 \mathrm{Nef}$ potently induces apoptosis in primary human brain microvascular endothelial cells via the activation of caspases. J Virol. 2005;79(7):4257-4269.

45. Lenassi M, Cagney G, Liao M, et al. HIV Nef is secreted in exosomes and triggers apoptosis in bystander CD4+ T cells. Traffic. 2010;11(1):110-122.

46. Duffy P, Wang X, Lin PH, et al. HIV Nef protein causes endothelial dysfunction in porcine pulmonary arteries and human pulmonary artery endothelial cells. J Surg Res. 2009; 156(2):257-264.

47. Mandell CP, Reyes RA, Cho K, et al. SIV/HIV Nef recombinant virus (SHIVnef) produces simian AIDS in rhesus macaques. Virology. 1999;265(2):235-251.

48. Almodovar S, Simonneau G., Humbert M, et al. Association between HIV-1 Nef signature sequences and HIV-related pulmonary hypertension. Poster presented at: American Thoracic Society International Conference; May 16-21, 2008; Toronto, Canada.

49. Petrosillo N, Chinello P, Cicalini S. Pulmonary hypertension in individuals with HIV infection. AIDS. 2006;20(16): 2128-2129. 